\title{
Review: newer and older antidepressants are effective in primary care and elderly patients with depressive disorders
}

Williams JW Jr, Mulrow CD, Chiquette E, et al. A systematic review of newer pharmacotherapies for depression in adults: evidence report summary. Ann Intern Med 2000 May 2;132:743-56.

QUESTION: In patients with depressive disorders, what is the effectiveness of newer antidepressants compared with placebo, older antidepressants, and each other? This abstract reports on the primary care and elderly patient subgroups in which newer and older antidepressants and placebo were compared.

\section{Data sources}

Studies were identified by searching the specialised clinical trial registry of the Cochrane Collaboration's Depression, Anxiety, and Neurosis Group (containing trials from such sources as Medline, EMBASE/Excerpta Medica, PsycLIT, LILACS, Psyndex, SIGLE, CINAHL, and Biological Abstracts from 1980 to January 1998); scanning the references of pertinent meta-analyses; consulting experts; and contacting pharmaceutical companies.

\section{Study selection}

Studies were selected if they were randomised controlled trials (RCTs) of $\geq 6$ weeks duration that compared a newer antidepressant with a newer or older antidepressant, placebo, or a psychosocial intervention; included patients with depressive disorders; and had a clinical outcome.

\section{Data extraction}

Data were extracted by 2 independent reviewers on patients; diagnoses; study design, including randomisation and blinding; intervention; and clinical outcomes. The primary outcomes were symptomatic response rate ( $\geq 50 \%$ improvement in symptoms as assessed by a depression rating scale or much or very much improved on a global assessment scale), dropouts, and discontinuation because of adverse events.

\section{Main results}

315 RCTs met the inclusion criteria. $90 \%$ of the trials were $6-8$ weeks in duration. Overall, similar response and total discontinuation rates existed for newer and older antidepressants. 27 RCTs evaluated 5540 primary care patients (mostly excluding patients with serious concomitant medical illnesses). Newer antidepressants were more effective than placebo for response rate (relative benefit increase [RBI] 60\%, 95\% CI 20\% to $110 \%$ ). Dropouts because of adverse events occurred in $2 \%$ of placebo, $8 \%$ of newer agents, and $13 \%$ of tricyclic antidepressant recipients. 27 RCTs involved 3929 patients $>60$ years of age. For response rate, newer antidepressants were more effective than placebo (RBI $40 \%$, CI $20 \%$ to $60 \%$ ) and similar to older antidepressants (RBI $0 \%$, CI $-10 \%$ to $10 \%$ ). Overall dropout rates and dropouts because of adverse events did not differ between newer and older antidepressants.

\section{Conclusions}

In patients with depressive disorders, newer antidepressants are more effective than placebo and are similar in effectiveness to older antidepressants. Primary care and elderly patients have a 40-60\% improvement in depressive symptoms with newer antidepressants compared with placebo.

\section{COMMENTARY}

This review by Williams et al confirms the equal efficacy of antidepressants, with similar dropout rates, irrespective of drug choice. It also refutes the common belief that few data are relevant to elderly and primary care patients because large randomised samples exist for each. Does the review help our assumption in geriatric psychiatry that tricyclics should not be used because newer drugs are safer? Does it also suggest that the primary care cohort mirrors the secondary researched population? Perhaps.

The included studies concentrated on short term measures of efficacy, yet depression affects social function, cognition, and quality of life, as well as classical symptoms. ${ }^{1}$ Few data exist about these important outcomes, posing pragmatic difficulties in using the review.

Are these the right outcomes and experimental designs for these populations? What does a "dropout" represent and are the reasons the same in older patients? What is the effect of differing dosing schedules, which add to the complexity of treatments? Finally, the adverse event rates need to be placed in a person specific context because people with certain treatments or conditions may have problems with tricyclics. This is borne out by the data, which suggest that the newer treatments are as effective as tricylics, but have fewer adverse events in the elderly.

This is a valid meta-analysis of the current evidence, yet it is also an indicator of the need for further research into depression. It is a helpful indicator that newer drugs should be first line in the elderly, especially when medical co-morbidity and potential for drug interactions exist, but the evidence is less convincing in younger patients. Studies with more clinically relevant outcome measures, especially of cognition, may inform this view. Full agreement on best practice in depression continues to require further work; considerable uncertainty is reflected by the fact that $>40$ treatment guidelines already exist in the UK alone. ${ }^{2}$

Roger Bullock, MA, MBBS Victoria Hospital Swindon, UK

1 Healy D, McMonagle T. The enhancement of social functioning as a therapeutic principle in the management of depression. J Psychopharmacol 1997;11:S25-31.

2 Littlejohns P, Cluzeau F, Bale R, et al. The quantity and quality of clinical practice guidelines for the management of depression in primary care in the UK. Br J Gen Pract 1999;49:205-10. 